\title{
Optokinetic Nystagmus: Modeling the Velocity Storage Mechanism
}

\author{
Claudio Maioli \\ Istituto di Fisiologia dei Centri Nervosi-C.N.R., 20131 Milano, Italy
}

To account for the oscillatory behavior of the optokinetic after-nystagmus (OKAN), a nonlinear model of the optokinetic system is proposed here that includes 2 first-order storage elements interconnected in a negative feedback loop. The adequacy of the model is tested by comparing its predictions with experimental data available in the literature. In addition, the question of the contribution of the storage element responsible for secondary OKAN (OKAN II) to the dynamic properties of the optokinetic nystagmus (OKN) is addressed.

The results show that the model is compatible with all modifications of the OKAN time course observed under various experimental situations. By comparing computer simulations and experimental data, it is inferred that (1) the dynamic properties of the optokinetic system during OKN and during OKAN are different; (2) the switching in velocity storage dynamics is not determined by the light-dark transition, but is induced whenever nystagmic slow phase velocity (SPV) is not sustained by an appropriate retinal slip error signal; (3) although no signs of adaptation are seen during OKN, the storage element responsible for OKAN II becomes charged during optokinetic stimulation; and (4) the time constants of the integrators are affected by the parameters of the preceding optokinetic stimulation.

It is known that the optokinetic nystagmus (OKN) elicited by a moving full-field visual pattern does not stop abruptly when the light is turned off, but slowly decays in the dark, giving rise to an optokinetic after-nystagmus (OKAN I) (Ohm, 1927; Ter Braak, 1936). OKAN I is often followed (almost always in some species) by a secondary after-nystagmus (OKAN II) beating in the opposite direction (Aschan and Bergstedt, 1955; Morimoto et al., 1963; Koerner and Shiller, 1972). Further nystagmus reversals can also be observed (Koerner and Shiller, 1972; Büttner et al., 1976; Maioli and Precht, 1984).

OKAN I is generally attributed to the discharge of a storage element residing in the brain stem that has become gradually charged during optokinetic stimulation (Cohen et al., 1977; Robinson, 1977). Similarly, OKAN II would be generated by a second, opposing storage element (also charged by the stimulus), with a lower gain and a longer time constant than that of OKAN I (Brandt et al., 1974; Büttner et al., 1976; Waespe and Henn, 1978; Waespe et al., 1978; Koenig and Dichgans, 1981). The 2

\footnotetext{
Received Feb. 6, 1987; revised July 21, 1987; accepted Aug. 21, 1987.

I wish to thank Professor C. Terzuolo for his suggestions and comments on this manuscript.

Correspondence should be addressed to Dr. Maioli, Istituto di Fisiologia dei Centri Nervosi-CNR, Via Mario Bianco 9, 20131 Milano, Italy.

Copyright (C) 1988 Society for Neuroscience $0270-6474 / 88 / 030821-12 \$ 02.00 / 0$
}

elements would work antagonistically, leading, after stimulus cessation, first to OKAN I and then to OKAN II. No satisfactory explanation for further nystagmus reversals has been proposed so far. It is generally accepted that OKAN simply results from the summation of the outputs of the 2 storage elements (Brandt et al., 1974; Büttner et al., 1976). Furthermore, similarities between the time course of postrotatory vestibular nystagmus and OKAN, and the modalities of their interaction, strongly suggest that the 2 storage elements are charged by both vestibular and optokinetic inputs (Ter Braak, 1936; Raphan et al., 1977; Robinson, 1977; Koenig and Dichgans, 1981).

The view that OKAN is generated by 2 storage elements that charge and discharge independently is based mainly on the observation that OKAN I and II are not influenced in the same manner by previous optokinetic stimulations. Actually, OKAN I and II are affected by the preceding stimuli in a complex way, their behavior often being reciprocal. Thus, an enhancement of OKAN II is generally accompanied by a shortening of OKAN I, as after prolonged stimulus duration (Brandt et al., 1974; Büttner et al., 1976), repetitive stimulation (Waespe and Henn, 1978), and brief visual fixation periods during OKAN I (Waespe et al., 1978). Furthermore, the idea of 2 independent storage elements is also supported by the clinical observation that, in patients with bilateral labyrinthine lesions, OKAN II can be retained despite a complete loss of the slow OKN component, and consequently of OKAN I (Zee et al., 1976).

Unfortunately, this conceptual model, although appealing for its simplicity, predicts a time course for OKAN different from that observed experimentally. In fact, if OKAN I and II were determined by the discharge of 2 independent and opposing storage elements of the first order, i.e., leaky integrators (Koenig and Dichgans, 1981), the time course of slow phase velocity (SPV) during OKAN would be described by the difference between 2 exponentials with real time constants. However, such a mathematical function gives a poor fit of the SPV profile of OKAN in the cat, which can, instead, be fitted by a damped sine wave (Maioli and Precht, 1984). Thus, the optokinetic system discharges in the dark with the typical oscillatory behavior of a second-order underdamped system.

Another unresolved issue is the contribution of the storage element responsible for OKAN II to the dynamic properties of OKN. Although there is convincing experimental evidence that this element is also charged during the optokinetic stimulation, and that OKAN II does not merely represent a central response to the occurrence of OKAN I (Brandt et al., 1974; Waespe et al., 1978), it is not clear whether the time course of the SPV buildup during $\mathrm{OKN}$ is compatible with a second-order storage mechanism whose dynamic properties are the same as those observed during OKAN.

In this paper, an attempt will be made to clarify these unsolved 


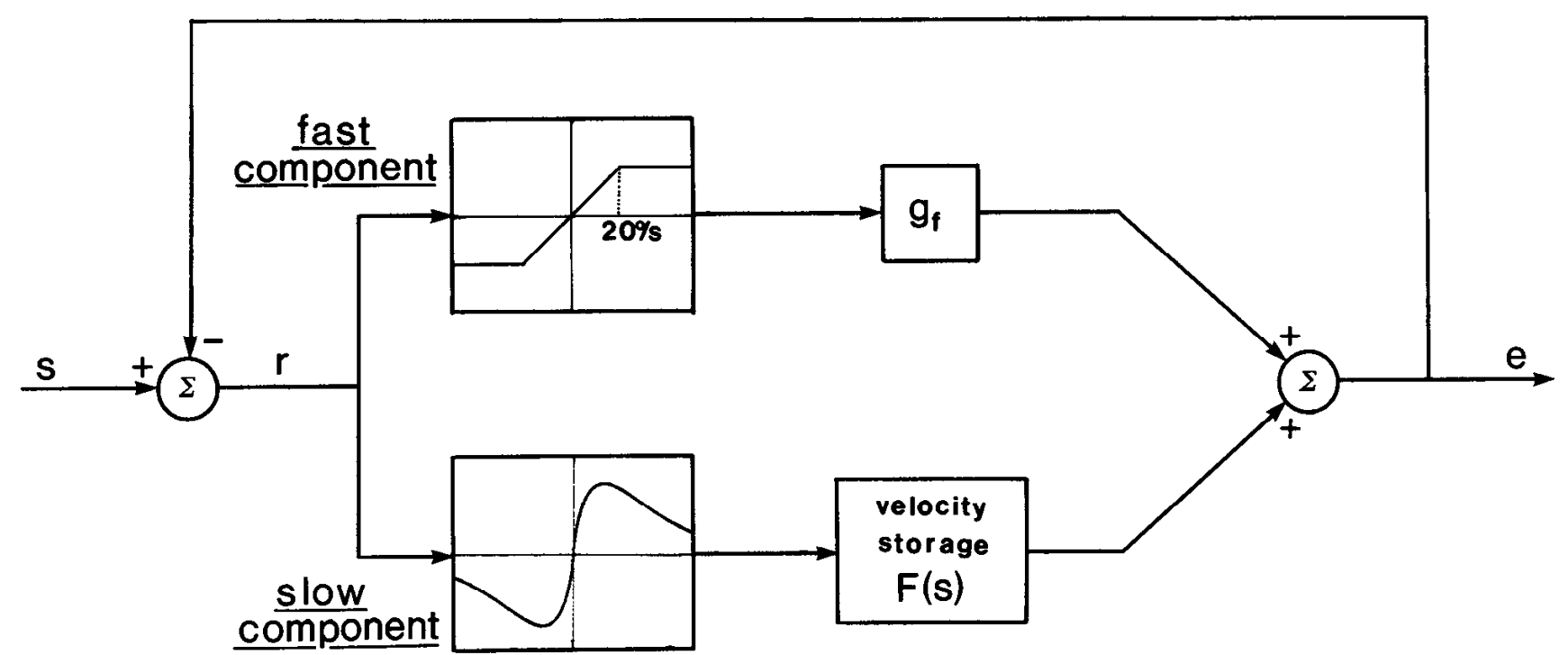

Figure 1. Simplified model of the optokinetic system. Nonlinear operators, identified from experimental data, are included in both the fast- and in the slow-component pathways. The slow-component nonlinearity is shown in detail in Figure $2 . s$, Visual world velocity; $r$, retinal slip velocity; $e$, eyc vclocity.

issues regarding the functional organization of the optokinetic storage mechanism. Since we are dealing with a nonlinear system, the best way to verify the adequacy of the assumptions is to formulate a model by which these assumptions can be rigorously tested by computer simulation. In this respect, one should note that several models of the optokinetic system and of its interactions with the vestibular system have been proposed in the last decade (e.g., Robinson, 1977; Raphan et al., 1979; Lisberger et al., 1981; Buizza and Schmid, 1982), and that they simulate quite accurately a large amount of data. However, all these models focus only on OKAN I and no attempt has been made to account for the presence and behavior of OKAN II.

\section{Materials and Methods}

Model predictions were obtained by computer simulation and were compared to the actual behavior of optokinetic responses, as derived from the literature. Moreover, previously reported experimental data (Maioli and Precht, 1984) were further elaborated to ascertain features of the optokinetic system that were deemed essential for developing the model. The techniques used in those experiments to record eye movements were fully described by Maioli and Precht (1984). Briefly, horizontal eye movements were recorded in 9 adult cats by DC electrooculography with chronically implanted electrodes. Recordings were performed with the head immobilized and after administration of amphetamine sulfate $(0.5 \mathrm{mg} / \mathrm{kg})$ to keep cats alert. Optokinetic stimulation was obtained by rotating around the animal a full-field high-contrast random-dot pattern (all elements $5^{\circ}$ wide). Details on the procedures for fitting the SPV profiles during OKAN can be found in Maioli and Precht (1984).

\section{Results}

Figure 1 provides a schematic representation of the current views on the organization of the optokinetic system. This can be considered a velocity servo aimed at reducing as much as possible the slip velocity of the visual word image on the retina. The overall optokinetic response is thought to be made up of 2 components, with different properties and distinct neuronal pathways. The "fast" component, responsible for quick changes in SPV following variations in stimulus velocity, can be suitably approximated by a pure gain element without dynamics. In contrast, the "slow" component, responsible for the generation of the SPV buildup to steady state in response to constantvelocity stimuli, results from a velocity storage element that, after stimulus cessation, gives rise to a slowly decaying OKAN. As indicated by the time course of OKAN in the dark, this element would have at least second-order dynamics (Maioli and Precht, 1984).

The nonlinearities of the system were modeled as follows: A simple saturation element was included in the "fast"-component path. The break point of the saturation curve varies between different species, and it is given by the stimulation velocity at which the initial jump in SPV (Cohen et al., 1977; Maioli and Precht, 1984), observed in response to velocity steps, reaches its maximum amplitude. In the cat this occurs at about $20 \%$ sec. As for the nonlinearity of the component path, it can mostly be ascribed to the nonlinear characteristics of detection of the visual image slip at the retinal level (Oyster et al., 1972; Collewijn, 1975; Cazin et al., 1980a; Hoffmann and Schoppmann, 1981; Hoffmann and Huber, 1983). The shape of this nonlinearity (Fig. 2) has been obtained by fitting the relationship between average SPV and retinal slip velocity when OKN has reached steady state (Maioli and Precht, 1984). Specifically, the function used is $A r^{b} e^{-r c}$, where $r$ is the retinal slip velocity and $A, b$, and $c$ are least-square estimates. The choice of this function has no theoretical significance and is only suggested by the data. Other functions could have been used, since the basic model predictions are largely insensitive to the specific form of the nonlinearity, provided that it has a maximum for a retinal slip of about $20 \% \mathrm{sec}$ and decays slowly thereafter. However, the chosen function also provides a good fit to some electrophysiological data. Indeed, the curve shown in Figure 2 is strikingly similar to the relationship, in the alert cal, between stimulation velocity and neuronal firing in both the nucleus of the optic tract (Hoffmann and Huber, 1983) and the vestibular nuclei (Keller and Precht, 1979) under open-loop conditions. These 2 nuclei are known to be important relay stations of the pathway conveying the "slow" OKN component. The vestibular nuclei data (Keller and Precht, 1979) are shown by asterisks in Figure 2 (after scaling) to allow a comparison. 


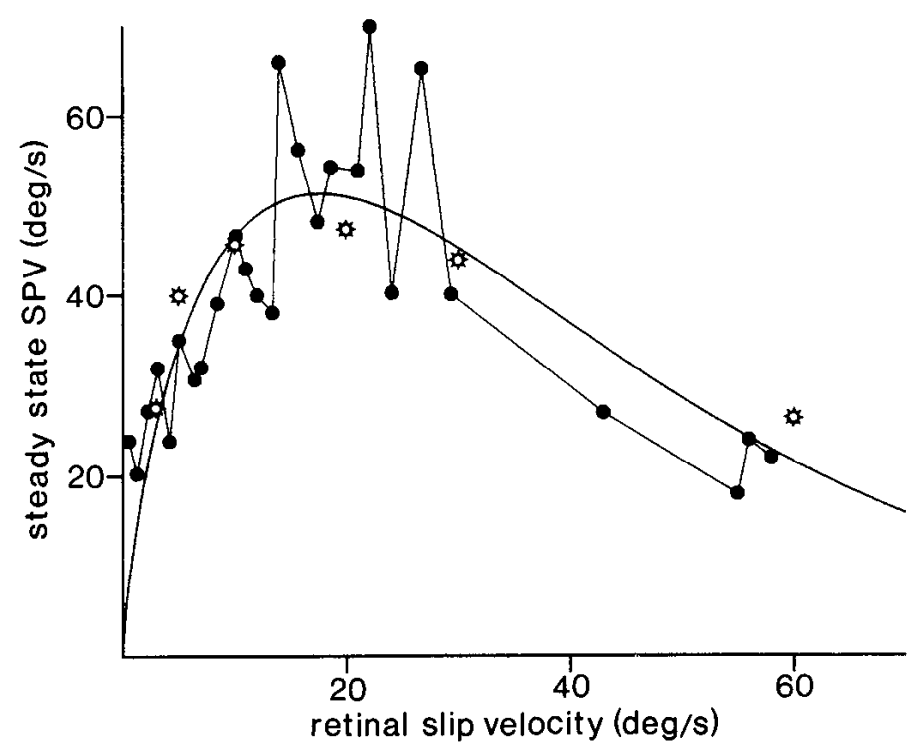

Figure 2. Nonlinearity of the slow-component pathway. The experimental data points (filled dots) describing the relationship between steadystate SPV and retinal slip velocity are fitted with the function $A r^{t} e^{-r c}$ (solid line), where $r$ is the retinal slip velocity and $A, b$, and $c$ are leastsquare estimates. Asterisks are scaled data from Keller and Precht (1979), and correspond to the average firing of vestibular nuclear neurons.

With this background, we can now approach the problem of characterizing the velocity storage of OKN. Obviously, the solution is not straightforward since the nonlinearities present in the retinal slip detection mechanism do not allow a description of the dynamics of the velocity storage during optokinetic stimulation by using SPV buildup. Thus, the following procedure will be used. First we shall see whether it is possible to derive the dynamic properties of the velocity storage during OKN from the time course of its discharge in the dark, i.e., from OKAN. Since this will turn out not to be the case, a model based on a second-order oscillating system composed of 2 leaky integrators will be developed and included in the framework of Figure 1. Inference with the interactions between the 2 integrators during OKN and OKAN will then be carried out by comparing model predictions with the known changes of OKAN I and II under specific experimental conditions.

\section{Velocity storage during $O K N$ and $O K A N$ has different dynamics}

If velocity storage behavior were the same both in the light and in the dark, its transfer function (and thus its impulse response) could be obtained from the SPV time course of OKAN. In fact, OKAN can be considered the open-loop discharge of the velocity storage charged to some level during the stimulation period. Since the OKAN profile can be accurately described by a damped sine wave, the velocity storage has to be modeled as a second-order underdamped system. The impulse response of such a system is

$$
e(t)=A e^{-T} \sin (\Omega t+\Phi)
$$

where $e$ is the eye velocity, $A$ a gain constant, $T$ a damping constant, $\Omega$ the oscillation frequency, and $\Phi$ the phase shift. Such impulse response can be computed for each recorded OKNOKAN sequence (which will be referred to as OKN trial) by estimating the parameters that best fit the OKAN time course.
The hypothesis that the dynamic properties of the velocity storage during both its charge and discharge are the same can then be tested by comparing the SPV profile recorded during OKN buildup with that predicted by the model, using the velocity storage impulse response derived from the OKAN time course. Because of the nonlinear characteristics of the retinal slip detection (Fig. 2), the model prediction of the OKN buildup is obtained by computing the convolution integral of the impulse response and the output of the "slow"-component nonlinearity.

A comparison between the computer simulation and actual data for a representative OKN trial is shown in Figure 3. Dots are samples of actual SPV taken every 2 sec during OKN and every 1 sec during OKAN. This particular trial, which illustrates the response to a $50^{\circ} / \mathrm{sec}$ stimulus, was chosen to dramatize the discrepancy between the data and the prediction of the model when one assumes the same dynamics for both the charge and discharge of the velocity storage. Specifically, the discrepancy concerns the SPV buildup during OKN. Given the large OKAN II and the fact that the steady-state gain is considerably less than one (see below), the model would in fact predict (Fig. 3, thick line) a steeper OKN rise time than the one actually observed, and a clear overshoot of the steady-state value. (The OKN overshoot is defined as the percentage amplitude by which the simulated SPV exceeds the steady-state value.)

The mismatch between actual OKN buildup and that predicted by the model was found very consistently in all the OKN trials studied. However, because of the nonlinear characteristics of the model, the extent of the overshoot not only is determined by the parameters of the estimated impulse response, but also depends strongly on the steady-state closed-loop gain, and thus on stimulus velocity. In fact, because of the nonlinearities in the retinal slip detection mechanism (Oyster et al., 1972; Hoffmann and Schoppmann, 1981), the OKN steady-state gain is high with slow stimuli and decreases progressively by increasing stimulus velocity. This dependency of OKN overshoot on closedloop gain is shown in Figure 4, where the ratio between OKN overshoot and OKAN II amplitudes is plotted against the steadystate closed-loop gain. The data points refer to 46 trials in 9 cats. In this sample, the maximum OKAN II amplitude was, on average, $19.8 \%$ of the steady-state SPV. For each simulation, the open-loop gain of the model was adjusted in order to match the experimentally found steady-state closed-loop gain. No "fast" OKN component was added to the model output since, for the purpose of this simulation, its effect would be negligible (its magnitude being quite small in the cat). The simulation shows quite clearly that if system dynamics were the same during SPV buildup as during OKAN, SPV would overshoot the steady state by more than $10 \%$ of the maximum OKAN II amplitude in all those trials in which the steady-state gain was less than 0.95 . However, no overshoot was ever detected even in those cases (i.e., when the gain was less than 0.9 ) where its theoretical amplitude should have been quite large. Furthermore, once steady state was reached, no sign of SPV adaptation was observed during constant-velocity stimulation lasting $1 \mathrm{~min}$ or more. Finally, in 4 cases the responses predicted by the model (open symbols, Fig. 4) largely overshot stimulus velocity, with an amplitude ratio between OKN overshoot and OKAN II larger than one. In 2 of these cases a damped oscillation, lasting for more than $3 \mathrm{~min}$, was present. Thus, the simulated responses are those of a system very close to instability. Note that also in these cases the real data did not show any overshoot in SPV.

The question is now, to what extent does the relationship 


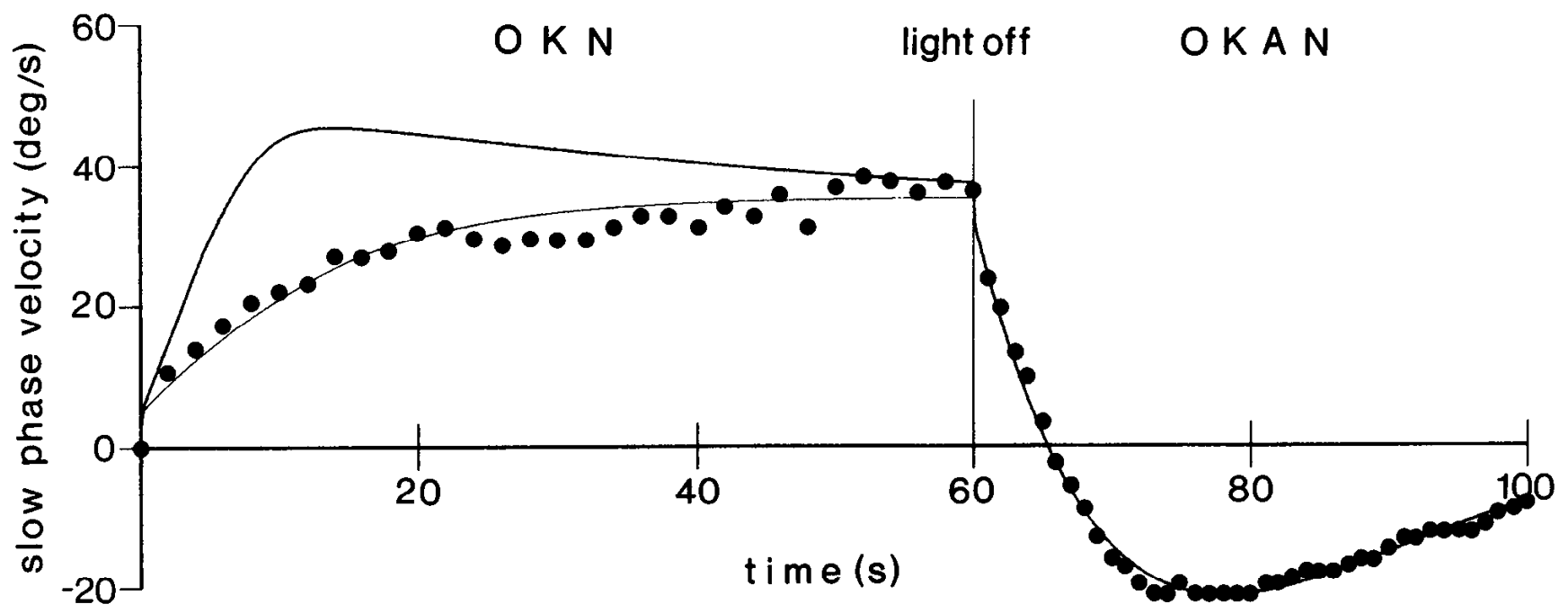

Figure 3. Computer simulations compared to sampled actual SPV (dots) in response to a constant-velocity stimulation of $50^{\circ} / \mathrm{sec}$. The stimulus lasts for $60 \mathrm{sec}$; thereafter the animal is kept in total darkness. The thick line is the computer simulation making the assumption that the velocity storage has identical dynamics during OKN and OKAN. The thin line is the model prediction of SPV buildup, assuming that no adaptation is occurring during OKN (see text).

shown in Figure 4 depend on the specific shape of the nonlinearity included in the model, this choice being somewhat arbitrary (see above)? In a closed-loop linear system, the amount of overshoot would be dependent on the steady-state gain. This relationship is given by the continous line in Figure 4. Here the parameters of the storage element were derived from the mean values of the experimental data $\left(T=0.069 \mathrm{sec}^{-1} ; \Omega=0.076\right.$ $\mathrm{rad} / \mathrm{sec} ; \Phi=2.032 \mathrm{rad}$ ) (Maioli and Precht, 1984). Up to a steady-state gain of about 0.98 the overshoot amplitude is more than $10 \%$ that of OKAN II, which is itself $14.2 \%$ of the steadystate level $\left(15^{\circ}-75^{\circ} / \mathrm{sec}\right)$. When the nonlinearity of Figure 2 is included in the model, one finds that its effect is conspicuous with respect to the SPV profile during the transient part of the response, but extremely small with respect to the overshoot amplitude. The dashed line in Figure 4 represents the simulated output of the nonlinear model (for the same average parameters as above) in response to a constant-velocity stimulation (in this instance $40 \% \mathrm{sec}$, the results being similar for other stimulation velocities). The predicted overshoot is indeed slightly larger than that obtained without the nonlinearity. Therefore, it is very unlikely that the lack of SPV overshoot at the end of OKN buildup is due to the nonlinear internal representation of retinal slip velocity. In fact, the magnitude of the predicted overshoot is only slightly modified, compared to that of a linear system, by including the highly nonlinear gain element of Figure 2 .

We conclude that the dynamic properties of the slow OKN component during visual stimulation and during its discharge in the dark are different.

\section{Velocity storage modeling}

From what we have seen so far, a model capable of simulating satisfactorily the behavior of the OKN storage mechanism should account for at least 2 basic facts: (1) the velocity storage discharges in the dark as a second-order underdamped system; (2) a difference exists between the dynamic properties of the slow OKN component during optokinetic stimulation and during OKAN, so that no SPV overshoot occurs during OKN.

Concerning the first point, it can be demonstrated that if OKAN were the output of a second-order system composed of
2 distinct leaky integrators (the velocity integrator proper and an opposing integrator, to be called adaptor), the system would be underdamped only when the 2 integrators were exerting negative feedback action on each other (see Appendix). Figure 5 is a block diagram of such a model, which should be considered a detailed version of the "velocity storage" box of Figure 1. Note that Figure 5 does not present an actual neural wiring diagram. Rather, it represents an equivalent circuit in which the only assumed isomorphism with the actual neuronal network consists in that the 2 leaky integrators are separate. In Figure $5, r$ is the retinal slip velocity internal representation found at

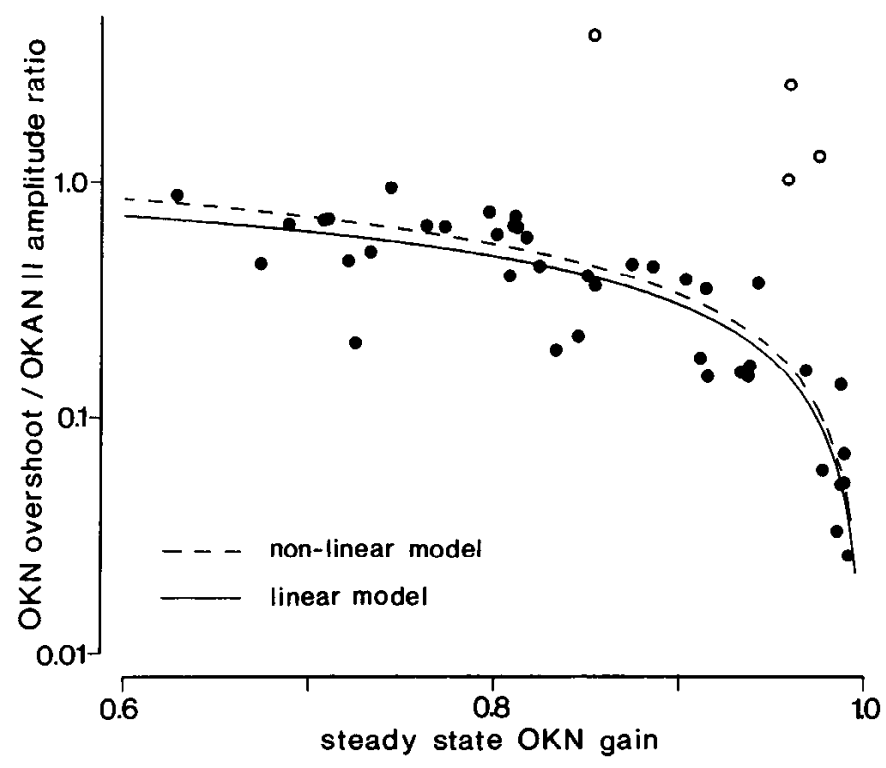

Figure 4. Predicted ratio between the amplitude of OKN overshoot and that of OKAN II as a function of steady-state OKN gain. Model parameters were computed by fitting the experimental SPV time course during OKAN. Dots indicate model predictions for individual trials ( $n=46$ in 9 cats). Open dots are those trials with predicted responses close to instability. The predictions of the model with the average OKAN parameters, including (dashed line) or not including (continuous line) the nonlinearity, are also plotted. 


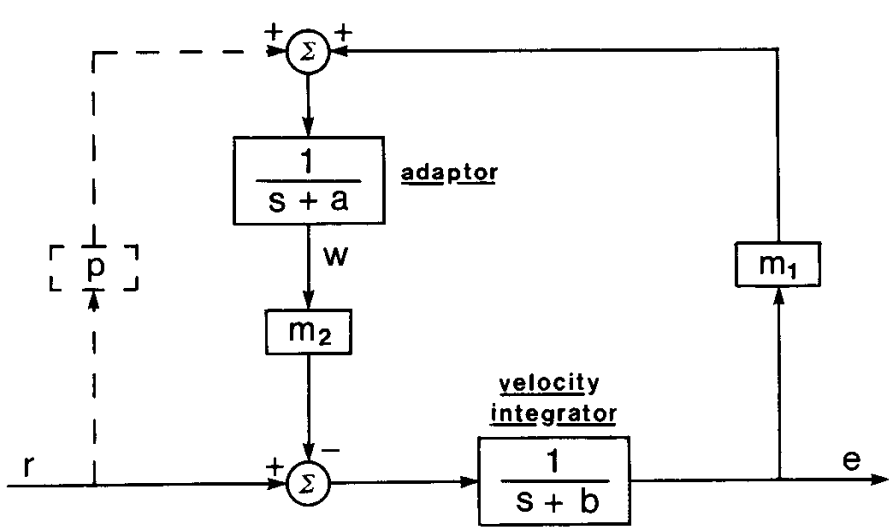

Figure 5. Block diagram of the model of the velocity storage. The model includes 2 leaky integrators (the velocity integrator and the adaptor) in a negative-feedback loop. Same conventions as in the Appendix, except that $p_{2}$ corresponds to $p$ and $p_{1}=1$. $r$, Internal representation of retinal slip velocity; $e$, eye velocity output of the velocity storage; $w$, output of the adaptor. A hypothetical path accounting for a direct charge of the adaptor by $r$ is indicated by the dashed line.

the retinopretectal level, $e$ is the eye-velocity command derived from the output of the velocity integrator, and $w$ is the output of the adaptor. The dashed line indicates the possibility that the adaptor is also charged directly by the retinal slip velocity signal through the gain element $p$. A more complete analysis of the model can be found in the Appendix.

We will now consider the second feature of the velocity storage behavior, namely, the lack of SPV overshoot during OKN. As stated previously, this behavior indicates that the dynamic properties of the system during OKN and OKAN are different. Various solutions can be proposed to account for this feature. However, they are not equivalent when the OKAN time course, after brief visual fixation periods, is also considered. Regarding this point, Waespe et al. (1978) have observed, both in monkey and in man, that brief visual fixation periods at the end of optokinetic stimulation considerably shorten OKAN I, while peak SPV of OKAN II gets larger and occurs earlier. This characteristic behavior is exactly what is predicted by the model of Figure 5, making the following assumptions: (1) the adaptor is charged to some extent during optokinetic stimulation, thus excluding the possibility that the lack of overshoot in SPV during OKN can be ascribed to the nonoperation of the adaptor during stimulation; (2) switching of parameter values between the 2 velocity storage conditions does not occur at the light-dark transition, but is induced whenever nystagmic SPV is not sustained by an appropriate retinal slip error signal, i.e., when the error becomes zero (as in the dark) or of opposite sign with respect to SPV. With this assumption, the velocity storage parameters during visual fixation and in the dark are the same.

The necessity of the first assumption can be demonstrated by studying model predictions while varying the level of charge reached by the adaptor at the end of OKN. Let us assume that $p=0$, which means that the adaptor is not charged directly by the retinal slip velocity signal. In this case, it can be demonstrated that model behavior in response to brief visual fixation periods during OKAN does not depend on the absolute level of adaptor charge, but rather is related to its charge in relation to the theoretical level that would be reached at steady state if the parameters of the model had the same values during both OKN and OKAN. Figure $6 A$ shows model predictions of the
SPV time course of OKAN following $5 \mathrm{sec}$ of visual fixation ( $s=0$ in Fig. 1), at the time indicated by the black strip below the time axis, as a function of the ratio $(h)$ between the actual adaptor level of charge at the end of OKN and the theoretical level that would be expected on the basis of $m_{1}$ and $a$ values compatible with the observed OKAN time course (see Appendix). Model parameters were adjusted to be consistent with the experimental average parameters describing the OKAN time course in the cat (dashed line). Accordingly, the fast OKN component gain $\left(g_{f}\right)$ was set to 0.3 . However, from a qualitative point of view, model predictions are basically unchanged for large variations of $g_{f}$. The open-loop gain was chosen to give a steady-state closed-loop gain of 0.9 with a stimulus velocity of $40 \%$ sec. The dotted line represents the response for $h=0$. The thicker line is the predicted response for $h=1$ (i.e., when model parameters are the same during both OKN and OKAN). The other simulated OKAN time courses in the visual fixation condition correspond to the indicated value $h$ of the initial charge of the adaptor. It can be readily observed that the model predicts that a short period of visual fixation during OKAN I inhibits OKAN I but enhances OKAN II, which starts and reaches peak amplitude earlier and attains higher SPV than in the control. Moreover, these features become more pronounced as the adaptor becomes more charged during OKN. Thus, qualitatively the model reproduces quite well the results obtained in monkey and in man by Waespe et al. (1978).

As for the second assumption, we can state that if parameter values were to change at the light-dark transition, there would be only 2 simple ways in which a modification of parameters could account for the lack of SPV overshoot during OKN, besides the one already excluded $\left(m_{1}-0\right.$ during optokinetic stimulation; dotted curve in Fig. $6 B$ ). The first would be if the adaptor was charged during $\mathrm{OKN}$, but its output was fed into the velocity integrator only in the dark $\left(m_{2} \approx 0\right.$ in the light). The second would be if during $\mathrm{OKN}$, the adaptor time constant was considerably shorter than that of the velocity integrator, so that the system became overdamped without overshooting steadystate SPV $\left[(a-b)^{2}>4 m_{1} m_{2}\right.$ and $\left.a>b\right]$ (see Appendix). Figure $6 B$ shows computer simulations of the model's behavior in response to $5 \mathrm{sec}$ of visual fixation in the early part of OKAN I (same conditions as in Fig. 6A), assuming that the change of model dynamics occurs at the light-dark transition and that $h$ is equal to one. When $m_{2}=0$ in the light, the effect of visual fixation is only a transient suppression of OKAN I (curve labeled $m_{2}=0$ ). This is due mainly to the fast OKN component. In fact, the discharge of the velocity integrator caused by the retinal slip input during visual fixation would have more or less the same effect that the negative feedback exerted by the adaptor has in the dark. Similarly, in case the system were to be overdamped in the light because of a large $a$ value (curve labeled "overdamped"), OKAN II would also be strongly reduced after fixation. The reasons for this behavior are 2 -fold: first, the steadystate charge level of a leaky integrator is directly proportional to its time constant $(1 / a)$, so that we fall to the condition of a low charge level of the adaptor at the end of OKN; second, during visual fixation, the adaptor follows the output of the discharging velocity integrator more quickly because of its short time constant. Even if we avoid the first disadvantage by coupling $m_{1}$ to $a\left(m_{1}=\right.$ constant $\left./ a\right)$ so that the steady-state charge level results are unaffected by changes in the integrator time constant [e.g., by setting the adaptor transfer function to $a /(s+$ a)], OKAN II becomes somewhat larger (curve labeled "over- 
damped*"). However, its absolute amplitude is much lower than that observed experimentally during the normal discharge of the velocity storage in the dark. One last point on this subject. Thus far it was assumed in the simulations that the adaptor was charged exclusively by the eye-velocity output signal, without receiving the retinal slip velocity signal as a direct input $(p=0$ in Fig. 5). However, the conclusions reached are also valid if this assumption is not true, i.e., if $p \neq 0$. Indeed, if the adaptor were to be charged directly by the retinal slip signal, OKAN II would be even more greatly reduced by visual fixation. Thus, this eventuality cannot be invoked to account for the observed OKAN II enhancement. Note also that irrespective of the assumptions made about the charge of the velocity storage, it is always possible to adjust the model parameters so as to obtain identical OKAN time courses in the absence of fixation suppression.

It could be argued that some ad hoc complex covariation of $m_{1}, m_{2}$, and $a$ at the light-dark transition could allow the model to simulate the responses observed experimentally. However, given our complete ignorance about the neuronal mechanisms underlying the velocity storage network, the assumption that system dynamics change whenever SPV is not sustained by an appropriate retinal slip signal seems most appealing because of its simplicity. In this case, the function of the adaptor would be to increase the speed with which the eye velocity command signal is reduced when the eye moves more quickly than the visual stimulus.

The model is also capable of simulating other effects of visual fixation on the OKAN time course. In particular, Waespe et al. (1978) have observed that OKAN II is temporarily inhibited during brief visual fixation periods, but recovers afterwards and slowly reaches a new maximum SPV value of somewhat less than $50 \%$ of the control value. As shown in Figure $6 C$, this behavior is in fact predicted by the model. The thick line without labeling is the computer simulation for a $3 \mathrm{sec}$ visual fixation during OKAN II, the assumptions being the same as for Figure $6 A$. Here the new peak SPV is about $40 \%$ of the control, but its amplitude can vary considerably as a function of the parameters of the damped sine wave, and it increases with the gain of the OKN "fast" component. The other curves represent simulations with different model features, labeled as in Figure $6 B$.

One last point should be considered. The predictions of the model when one assumes that the system has the same dynamics during both OKN and OKAN do not fit satisfactorily the actual SPV buildup. If, instead, we make the assumption that whenever SPV is not sustained by an appropriate retinal slip error signal, a change occurs in the strength with which the adaptor exerts a negative feedback action on the velocity integrator (i.e., in the value of $m n_{2}$ ), the predicted SPV profile during OKN (thin line in Fig. 3) is in good agreement with the data. This simulation was obtained by assigning to the model parameters the values obtained by fitting the OKAN time course $(h=1)$ and by setting $m_{2}=0$ during OKN. In general, the actual SPV buildup was rather well simulated by modeling the velocity storage during OKN as a simple first-order element with a time constant equal to that derived for the velocity integrator of Figure 5 by fitling the SPV time course during OKAN with a damped sine wave.

Figure 6. Simulations of SPV time course of OKAN in response to brief visual fixation periods. Dashed lines in all panels represent the

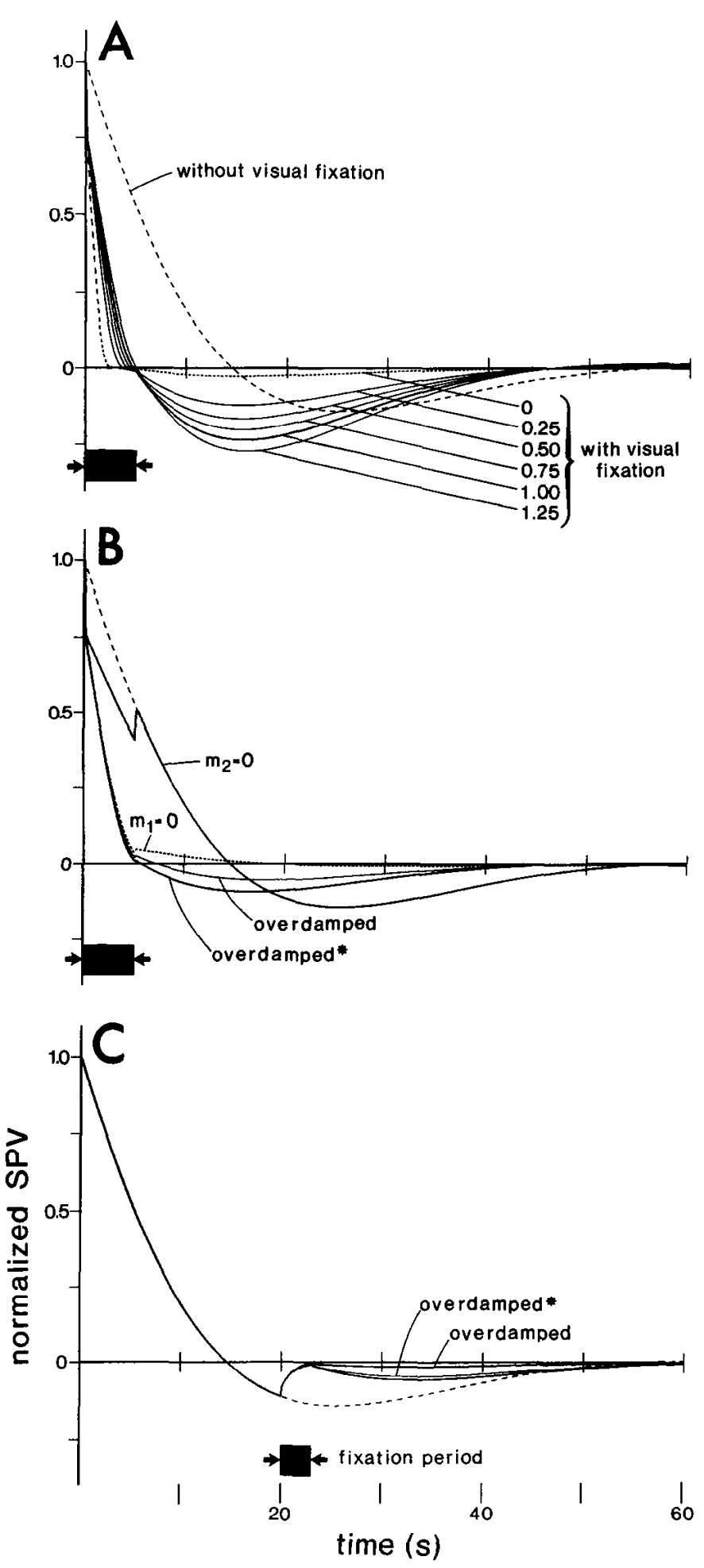

SPV time course in the absence of visual fixation. Black stripes below the time axes indicate the occurrence of the fixation periods. $A$, Model predictions following $5 \mathrm{sec}$ of visual fixation at the beginning of OKAN for the indicated initial charge levels of the adaptor, and assuming that the velocity storage has the same dynamics during fixation and in the dark. $B$, Same conditions as in $A$, but assuming that the change in velocity storage dynamics occurs at the light-dark transition. Labels indicate the differeent parametric conventions used for the charge of the velocity storage (see text). $C$, Model predictions with $3 \mathrm{sec}$ of visual fixation during OKAN II. The thick unlabeled line corresponds to the prediction, with the same assumptions as in $A$. The other curves represent simulations with different model features of the charge of the velocity storage, labeled as in $B$. 

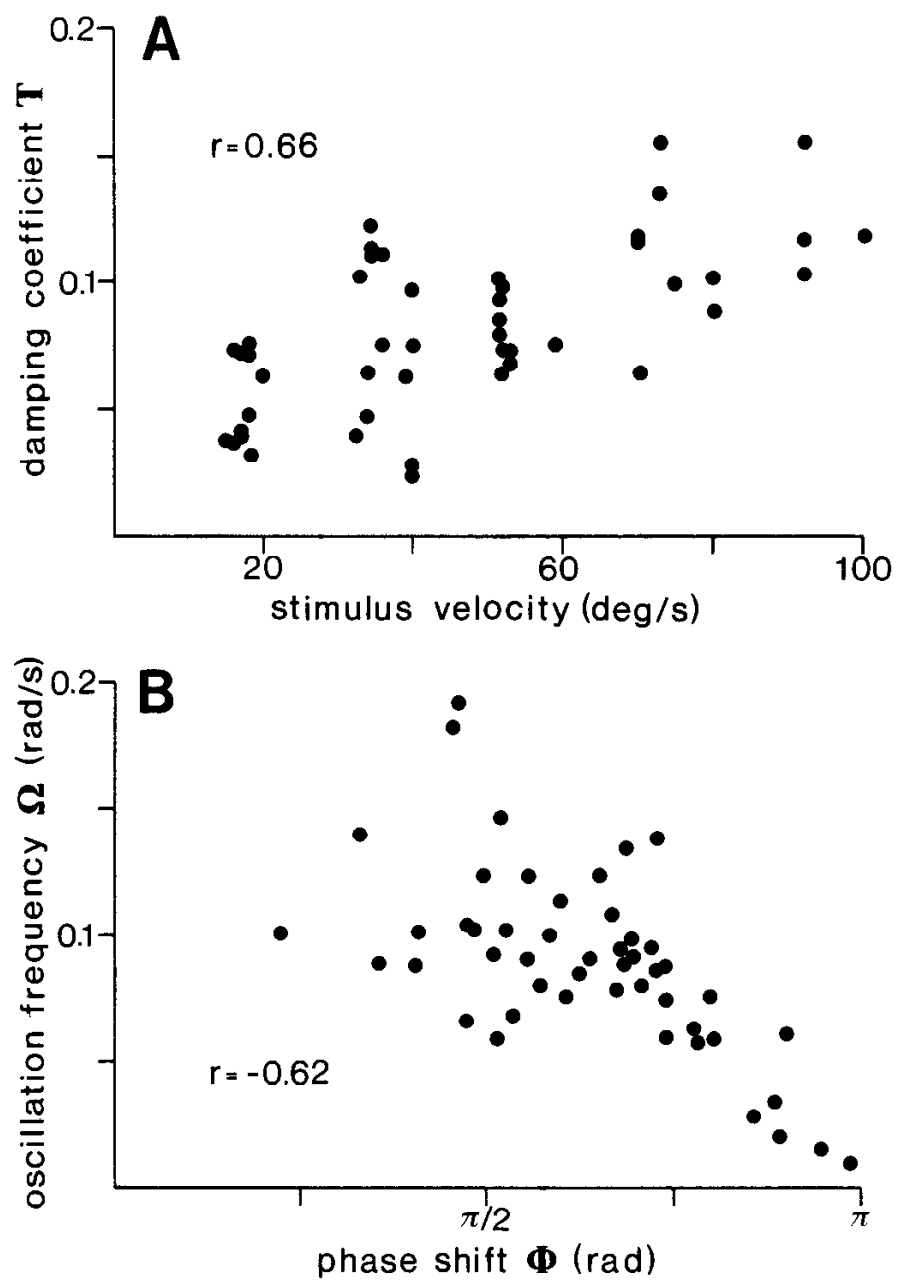

Figure 7. A, Relationship between the damping coefficient $T$ of the SPV time course of OKAN and stimulus velocity. $B$, Relationship between oscillation frequency $\Omega$ and phase shift $\Phi$ of the damped sine wave best-fitting OKAN time course.

\section{Velocity storage properties depend on stimulation conditions}

As mentioned above, prolonged optokinetic stimuli (Brandt et al., 1974; Büttner et al., 1976) and repetitive stimulations, i.e., sequences of constant-velocity stimuli at regular intervals (Waespe and Henn, 1978), induce in monkeys and humans a shortening of OKAN I and an enhancement of OKAN II. In the cat, an enhancement of OKAN II has also been observed after prolonged stimulation (Clément et al., 1981). In the case of repetitive stimuli, the changes in the OKAN time course are a clear indication that the conditions of stimulation may exert a parametric control on the velocity storage. Most likely, this is also the reason for the modifications of the OKAN time course observed after prolonged optokinetic stimuli. Indeed, these modifications cannot be attributed to the fact that the system is tested in a nonstationary state, because the duration of the stimulation used in the experiments (up to $15 \mathrm{~min}$ ) is far longer than the overall duration of OKAN or than the time necessary for a complete charge of the velocity storage (Cohen et al., 1977, 1981; Segal and Liben, 1985).

It is evident that it is quite easy to adjust the parameters of any model previously proposed in order to account for any desired change in the relative contributions of OKAN I and II to the overall OKAN. Moreover, this can be accomplished in a number of different ways. The question then becomes, is a particular solution suggested by available experimental data in the context of the specific model we have proposed?

A demonstration that stimulation parameters can influence the time constant of the integrators is provided by the presence of a significant correlation (Fig. $7 A$ ) between the damping constant $T$ of the SPV time course of OKAN and stimulus velocity $(r=0.656 ; p<0.001)$. Since $T$ is equal to the mean of $a$ and $b$ (see Appendix), this relationship indicates that adaptor and/ or velocity in tegrator time constants tend to decrease as stimulus velocity increases. This is also in keeping with the decrease in OKAN I duration caused by increasing the stimulation velocity, as observed in monkeys (Cohen et al., 1977). By contrast, neither $\Omega$ nor $\Phi$ was found to correlate with stimulus velocity, steadystate SPV, or retinal slip velocity of the preceding OKN.

A parametric change of the time constant of the integrators can also account for the variability of the OKAN time course observed among successive trials in the same animal. This "spontaneous" variability is characterized by a specific pattern of covariation among the parameters of the damped sinusoids fitting SPV profiles of OKAN. For instance, a significant correlation was found between frequency of oscillation $(\Omega)$ and phase shift $(\Phi), \Omega$ being inversely proportional to $\Phi(r=-0.624$; $p<0.001)$. This relationship is shown in Figure $7 B$, where all data points taken from 9 cats are plotted. An increase in the variability of $\Omega$ values occurs when $\Phi$ decreases. No correlation exists between the damping coefficient $T$ and $\Omega(r=0.129 ; p>$ 0.3 ). A weak but significant correlation was instead found between $T$ and $\Phi(r=0.458 ; p<0.01)$. Inspection of the equations relating the parameters of the damped sinusoid to those of the model [eqs. (7-9) in the Appendix] reveals that the observed relationships between the parameters of the OKAN time course can be accounted for only by assuming some covariation of the parameters of the model. Interestingly, probably the simplest covariation involves the integrators' time constants, which should change in a reciprocal manner in order to keep $T$ uncorrelated with $\Omega$ and $\Phi$. Indeed, with this latter constraint it would be rather difficult to account for the relationship of Figure $7 B$ without assuming a covariation of time constants. Confirmation that such a coupling between the integrators' time constants can mimic closely-using an appropriate parametric adjustmentthe relationship shown in Figure $7 B$ has also been obtained by computer simulation.

A covariation of the integrators' time constants, depending on the parameters of the previous optokinetic stimuli, could also account for the above-mentioned reciprocal changes of OKAN I and II experimentally observed in monkeys and humans after prolonged or repetitive stimuli (Brandt et al., 1974; Büttner et al., 1976; Waespe and Henn, 1978). The effect of reciprocal changes of $a$ and $b$ on the time course of OKAN is shown in Figure 8. Here, the values of $a$ and $b$ compatible with the average OKAN in the cat (thick line) were computed from the mean OKAN time course parameters by assuming $h=1$ (see Appendix). The values of the time constants of the velocity integrator $\left(\tau_{b}=1 / b\right)$ and of the adaptor $\left(\tau_{a}=1 / a\right)$ were 25.6 and $10.1 \mathrm{sec}$, respectively. Simulated OKAN time courses were then obtained by varying the parameters $a$ and $b$ by constant steps and keeping their sum equal to twice the average value of the sine wave damping constant $\left(T=\right.$ constant $\left.=0.069 \mathrm{sec}^{-1}\right)$. As a consequence, the various OKAN time courses have the same overall duration, but correspond to different relative contributions of OKAN I and II. Specifically, as $\tau_{a}$ gets larger and $\tau_{b}$ 
smaller, OKAN I becomes progressively shorter. At the same time, the SPV of OKAN II is enhanced and reaches its peak value faster. Thus, the proposed model can simulate the reciprocal changes in OKAN I and II found experimentally in monkeys and humans following prolonged stimulus durations (Brandt et al., 1974; Büttner et al., 1976) and repetitive stimulations (Waespe and Henn, 1978) without having to assume 2 independent storage mechanisms.

Finally, it should be noted that, contrary to what would be predicted according to this latter view, the time constant of the adaptor is not necessarily longer than that of the velocity integrator. Indeed, by assuming an $h$ value of about 1 , the majority of the OKAN time courses observed in the cat require a time constant for the adaptor that is shorter than that of the velocity integrator (for instance, as for the average OKAN time course; see above).

\section{Model predictions of $O K N$ nonlinearities}

The comparison between model predictions in response to various inputs and to actual experimental data proved to be satisfactory. However, because of the inevitable arbitrariness in the choice of nonlinear operators, it is important to consider in detail the predictions of the model concerning the nonlinear features of the experimental data. In the present context, the nonlinear features of $O K N$ have been attributed to a nonlinear retinal slip detection (Oyster et al., 1972). The causal relations between the latter and OKN nonlinearities have been discussed previously (Maioli and Precht, 1984; see also Demer, 1981; Buizza and Schmid, 1982; Waespe et al., 1983). However, by defining the characteristics of the velocity storage, it becomes possible to use computer simulation to quantitatively test these relationships.

It was shown in Figure 3 that the SPV buildup of the OKN can be satisfactorily simulated with a first-order storage mechanism, and that an additional pole has to be included in the model only when SPV is not sustained by an appropriate retinal slip. Using this assumption we can verify whether the model predicts all major nonlinearities observed in the $\mathrm{OKN}$.

Figure $9 A$ shows the relationship predicted between steadystate SPV and stimulus velocity. As observed experimentally (cf. fig. 5 in Maioli and Precht, 1984), the slope of the curve decreases as a function of stimulus velocity, indicating a gradual saturation of the response. This relationship is almost unaffected by the value of the time constant of the velocity integrator. By contrast, the predicted settling time of the SPV (the interval between the onset of the stimulus and the time at which SPV reaches $95 \%$ of the steady-state value) is an increasing function of stimulus velocity (Fig. $9 B$ ). This relationship is also observed in actual data (cf. fig. 4 in Maioli and Precht, 1984). Figurc $9 B$ illustrates the results of the simulation for different values of the time constants. Note that, as shown in the previous section, the time constant of the velocity integrator presumably tends to decrease as stimulus velocity increases.

A third nonlinear feature of the optokinetic system is found in the relationship between maximum SPV and stimulus amplitude for responses to sinusoidal stimuli. Specifically, gain decreases as stimulus velocity increases (see fig. 10B in Maioli and Precht, 1984). As shown in Figure $9 C$, this nonlinear relationship is also accurately predicted by the model. In Figure 9 (time constant $=8 \mathrm{sec}$ ), the gain was computed as the ratio between maximum SPV and maximum stimulus velocity, ir-

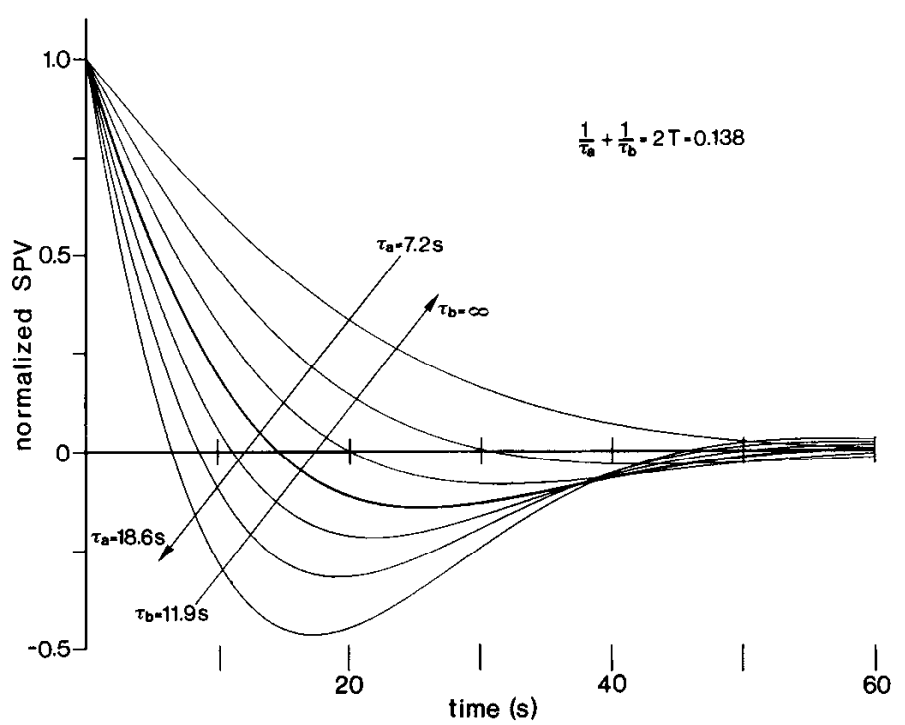

Figure 8. Effects of a reciprocal covariation of the time constants of the velocity integrator $\left(\tau_{b}\right)$ and of the adaptor $\left(\tau_{a}\right)$ on the OKAN time course. The reciprocals of $\tau_{a}$ and $\tau_{b}$ were varied by constant steps (equal to $0.015 \mathrm{sec}^{-1}$ ), keeping their sum equal to twice the mean damping constant $T$ of OKAN time course. The thick line represents the average time course of OKAN $\left(\tau_{a}=10.1 \mathrm{sec}\right.$ and $\left.\tau_{b}=25.6 \mathrm{sec}\right)$. The arrows point in the direction of increase of the corresponding parameters, whose range of variation is also indicated.

respective of the distortions present in the simulated responses with large stimuli.

Finally, the model also predicts (Fig. 9D) the characteristic distortion of the SPV profile that is experimentally observed in response to high-frequency, large-amplitude stimuli (cf. fig. 9 in Maioli and Precht, 1984). Note that the simulation of this distortion requires the presence in the model of the low-gain, fastcomponent pathway.

\section{Discussion}

In this paper, a simple model of OKN velocity storage has been derived from the basic observation that SPV decay during OKAN can be closely described by a damped oscillation (Maioli and Precht, 1984). The model consists of a negative feedback control system aimed at reducing steady deviations of the system output from a given reference level. This task is accomplished simply by subtracting from the input the low-pass-filtered system output. Low-pass filtering guarantees that only slow, long-lasting deviations from the set point are effectively reduced, these being considered as disturbances to the system. The velocity storage model was then included in a block diagram of the optokinetic system which was based on the main anatomical and functional concepts that have thus far been established. Furthermorc, nonlinear operators were introduced to account for the nonlinearities observed in the optokinetic responses.

Several experimental observations concerning the role of the vestibular nuclei in visual-vestibular interactions lend support to the view that the output of each of the 2 storage elements is fed to the input of the opposing leaky integrator. In fact, it is well known that postrotatory vestibular nystagmus (PRN) lasts longer than primary vestibular fibers discharge, indicating that for the vestibular system too a central storage mechanism must be postulated (Raphan et al., 1977; Robinson, 1977). In addition, a secondary PRN is almost always observed. The numerous features common to both OKAN I and primary PRN, as 

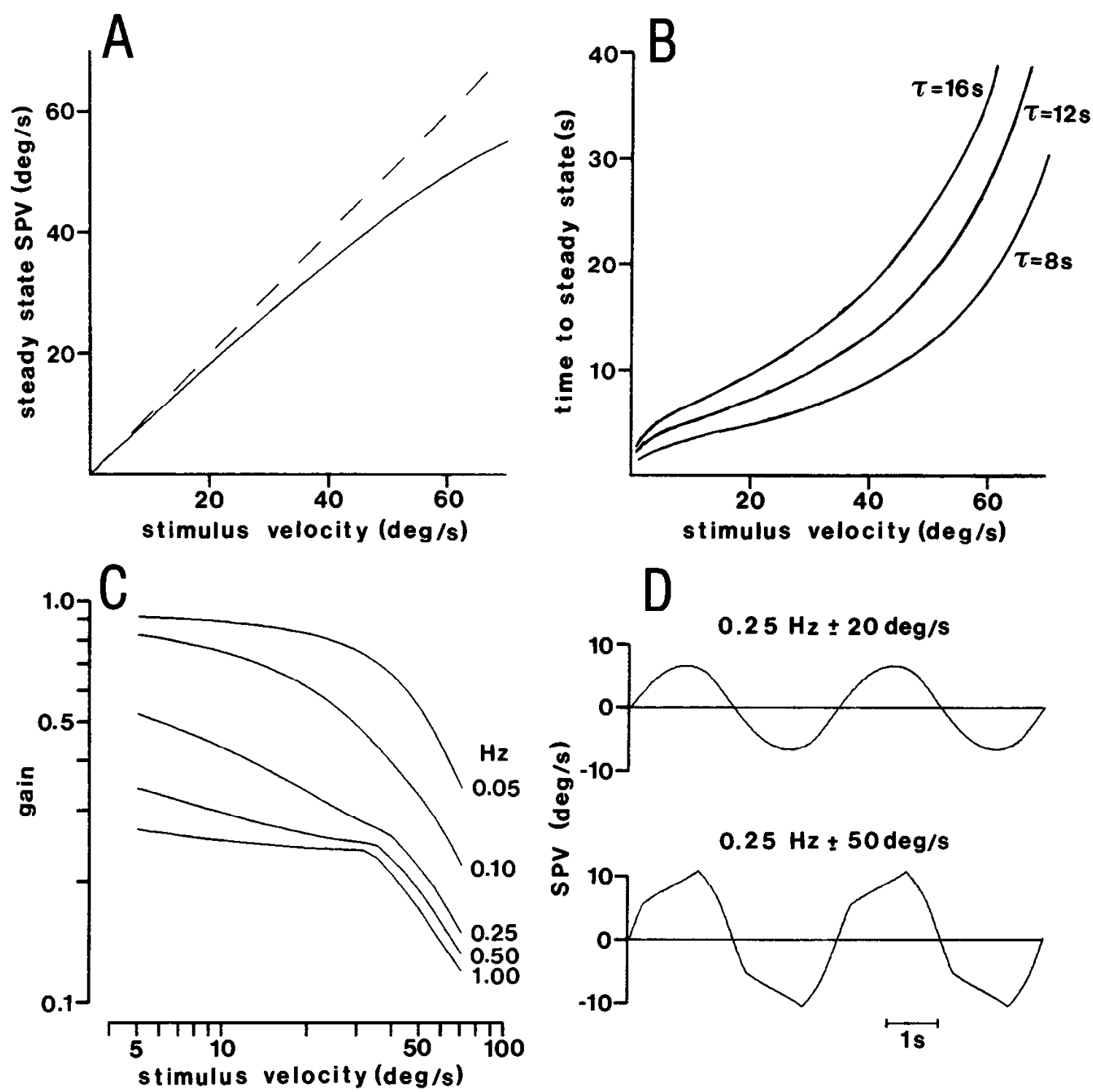

Figure 9. Model predictions of OKN nonlinearities. A, Relationship between steady-state SPV and stimulus velocity. The model predicts a gradual saturation of the responses. $B$, Relationship between settling time and stimulus velocity for different time constants of the velocity integrator. Settling time is an increasing function of stimulus velocity. $C$, Relationship between gain of sinusoidal responses and stimulus velocity at different frequencies. Gain is a decreasing function of stimulus velocity. $D$, SPV profile in response to sinusoidal stimuli. Large stimulus velocities induce a characteristic distortion of the SPV profile.

well as the way with which they interact following combined visual-vestibular stimulation, have suggested that both aftereffects depend on a common central storage mechanism, which would be activated by both optokinetic and vestibular stimuli (Ter Braak, 1936; Raphan et al., 1977; Robinson, 1977). Furthermorc, there are good arguments for assuming that the secondary PRN and OKAN II are also generated by a common central integrator (Koenig and Dichgans, 1981). This view is also supported by the finding that vestibular and optokinetic signals converge at the level of the vestibular nuclei (Dichgans et al., 1973; Henn et al., 1974; Allum et al., 1976; Waespe and Henn, 1977a; Keller and Precht, 1978; Cazin et al., 1980b), indicating that the 2 systems share a final common path. Finally, in the alert monkey and rabbit, activity of the vestibular nuclei was shown to parallel nystagmic SPV rather strictly during the primary and secondary phases of both PRN and OKAN (Waespe and Henn, 1977b; Buettner et al., 1978; Neverov et al., 1980). Thus, vestibular nuclei are the terminal site of primary vesti- bular afferents and, at the same time, convey the final eyevelocity command signal during both primary and secondary aftereffects. This means that the final output of the velocity storage is fed back to the input of both storage elements, thus accounting for potential oscillatory behavior. Since vestibular and optokinetic inputs share the same velocity storage mechanism, it must be concluded that these electrophysiological data can also account for the oscillatory behavior observed during OKAN.

It should be stressed again that the proposed model of the velocity storage must be interpreted as an equivalent circuit and not as a precise wiring diagram of how the 2 leaky integrators are interconnected. The important point is that the oscillatory behavior observed experimentally during OKAN can only arise if the outputs and the inputs of the 2 leaky integrators are fed reciprocally into each other. Consequently, in all cases, the behavior of the velocity storage is satisfactorily described by eq. (2) in the Appendix. It follows that the inferences regarding the 
functional organization of the velocity storage worked out in this paper are relatively independent of the specific neural implementation of the velocity storage. For instance, Leigh et al. (1981) have proposed a model of the optokinetic system that includes 2 storage elements reciprocally interconnected. The model was designed to simulate a pathological condition in which a spontaneous nystagmus periodically reverses direction (periodic alternating nystagmus), and no attempt was made to verify its adequacy in simulating normal OKAN under different experimental conditions. The pathological nystagmus results from an adaptation storage element that, under certain conditions, can render the system unstable. With some modifications and a proper parametric adjustment, the model can predict an OKAN time course that is described by a damped sine wave, and the above-considered modifications of OKAN induced in various experimental conditions. To the extent that the model by Leigh et al. (1981) embodies the same basic hypothesis of reciprocal interconnection, it can be considered an implementation of the abstract scheme proposed in this paper.

The main conclusions drawn from the comparison between the predictions of the present model and available experimental data are the following: (1) the 2 storage elements required for OKAN generation exert, at least in the dark, negative feedback on each other; (2) the dynamic properties of the optokinetic system during OKN and during OKAN are different; (3) although no signs of adaptation are seen during OKN, the adaptor becomes charged during optokinetic stimulation; (4) switching between the 2 velocity storage conditions is not determined by the light-dark transition, but is induced whenever nystagmic SPV is not sustained by an appropriate retinal slip error signal; and (5) the time constants of the integrators are affected by the parameters of the previous optokinetic stimulation.

Perhaps the simplest explanation of (2), above, is that the adaptor does not contribute to the dynamic properties of the slow optokinetic component during OKN. This may be the result of a very low $m_{2}$ value (see Fig. 5) during normal optokinetic stimulation, as suggested by the following observations: (1) the lack of overshoot in SPV during constant-velocity stimulation implies that the coupling between the velocity integrator and the adaptor is different during OKN and OKAN; (2) a satisfactory simulation of $\mathrm{OKN}$ buildup can be obtained with a simple first-order velocity storage; (3) OKAN II enhancement induced by brief visual fixation periods strongly suggests that the adaptor is charged during optokinetic stimulation. If so, the lack of SPV overshoot in the transient part of OKN cannot be due to the fact that the adaptor is inactive during stimulation. A similar boosting of secondary PRN has also been described (Collins, 1968; Koenig and Dichgans, 1981) following brief periods of visual fixation during primary PRN. In addition, it has never been shown that prolonged constant velocity optokinetic stimuli, while enhacing OKAN II, also determine a slow decrease of SPV during OKN. This would be expected if the interaction between the 2 storage elements did not change at the OKN-OKAN transition. On the contrary, $30 \mathrm{~min}$ of continuous stimulation induces in the cat a $30 \%$ increase of SPV during $\mathrm{OKN}$ and, after the light is switched off, a marked and longlasting OKAN II (Clément et al., 1981).

The notion of an adaptor that is charged by the optokinetic stimulus but has no effect on the oculomotor output raises the problem of the purpose of such an element. Indeed, it is hard to accept that the system is engaged in a buildup of neuronal activity without any apparent reason. A possible role for such a central adapting mechanism is suggested by the dissociation between $\mathrm{OKN}$ and self-motion perception (circularvection) during constant-velocity stimuli (Brandt et al., 1974). During prolonged stimulation, while the perceived velocity of induced circularvection decreases, the intensity of the ongoing nystagmus remains more or less unchanged. A reversal in the direction of self-motion perception can eventually be experienced. It should be noticed that no dissociation between the direction of subjective velocity and that of the oculomotor response is present during OKAN (Brandt et al., 1974). The decrease of self-motion perception can be taken as the expression of an adaptive process that develops during continous stimulation, even if it does not manifest itself at the oculomotor output. One could then postulate that both perceptual and oculomotor adaptations originate from a common storage mechanism that is charged during persistent stimuli (optokinetic and vestibular), but exerts variable and different controls on nystagmus and turning sensation, depending on stimulus conditions. Interestingly, during prolonged constant angular acceleration in the dark, the time courses of vestibular nystagmus and sensory experience are also different, adaptation occurring earlier in the latter than in the former (Guedry and Lauver, 1961; cf. Guedry, 1974). This fact results in the known difference between the slopes of nystagmus and sensation cupulograms, which has already led Groen (1961) to postulate the existence of a central inhibitory mechanism that suppresses sensation more strongly than nystagmus during longlasting vestibular stimuli.

We come now to the question of the functional significance of secondary nystagmus. As mentioned above, both OKAN II and secondary PRN have been interpreted as being the result of an adaptive homeostatic process aimed at counteracting a persistent unidirectional nystagmus (Young and Oman, 1969; Malcolm and Melvill-Jones, 1970; Brandt et al., 1974), such as that originating from a central imbalance of spontaneous input from the 2 labyrinths in various pathological conditions. The "repair" mechanism would also come into play in the case of prolonged constant stimuli, which seldom, if ever, occur naturally. In fact, since in the dark the brain has no way of distinguishing between a sustained vestibular stimulus and a pathological imbalance in the level of activity of vestibular nuclei, it may react to both in a similar manner by trying to readjust balance. However, when visual information is available, the system has the possibility of assessing whether or not the oculomotor output is the appropriate response to an external stimulus (optokinetic and/or vestibular). This can be done by comparing the direction of the compensatory eye movement with the direction of the residual retinal slip. If the directions are the same, the response is useful for stabilizing vision and must not be suppressed; if the directions are of opposite sign, as in the presence of a spontaneous nystagmus, the eye response is functionally deleterious and must be reduced. This can be achieved by varying appropriately the strength of the inhibitory coupling of the adaptor with the primary storage element. If the system were not able to discriminate between these 2 conditions, the adaptation process for maintaining homeostatic balance would also tend to reduce appropriate oculomotor responses in the light, especially when they are produced by head movements with low-frequency components.

One last point, concerning a clinical observation mentioned in the introduction, namely, that the complete loss of OKAN in human patients with bilateral labyrinthine lesions is not necessarily accompanied by a similar loss of OKAN II (Zee et al., 
1976). This finding is apparently in conflict with the model proposed here, since it seems to support the view that OKAN is generated by 2 independent storage elements. In a forthcoming paper (C. Maioli and W. Precht, unpublished observations), data from bilaterally labyrinthectomized cats will be used to demonstrate that this clinical observation can be reconciled with the model proposed here.

\section{Appendix}

The behavior of a second-order system composed of 2 first-order leaky integrators is described by

$$
\left[\begin{array}{l}
\dot{e}(t) \\
\dot{w}(t)
\end{array}\right]=\left[\begin{array}{cc}
-b & m_{2} \\
m_{1} & -a
\end{array}\right] \cdot\left[\begin{array}{l}
e(t) \\
w(t)
\end{array}\right]+\left[\begin{array}{l}
p_{1} \\
p_{2}
\end{array}\right] \cdot r(t)
$$

or, more concisely (with boldface type indicating matrices),

$$
\left[\begin{array}{c}
\dot{e}(t) \\
\dot{w}(t)
\end{array}\right]=\mathbf{A} \cdot\left[\begin{array}{c}
e(t) \\
w(t)
\end{array}\right]+\mathbf{P} \cdot \mathbf{r}(\mathrm{t})
$$

Here, $c(t)$ and $w(t)$ are the outputs of the leaky integrators (the velocity integrator and the adaptor, respectively), $r(t)$ is the input-forcing function, $a$ and $b$ are the reciprocals of the integrators' time constants, $m_{1}$ and $m_{2}$ are the coupling variables betwecn integrators, whilc $p_{1}$ and $p_{2}$ are constants (which may take a zero value) that weigh the charge of the 2 integrators by the external input $r(t)$.

The Laplace $s$-transform of equation (2) is

$$
s \cdot\left[\begin{array}{c}
e(s) \\
w(s)
\end{array}\right]-\left[\begin{array}{l}
e(0) \\
w(0)
\end{array}\right]=\mathbf{A} \cdot\left[\begin{array}{l}
e(s) \\
w(s)
\end{array}\right]+\mathbf{P} \cdot \mathbf{r}(\mathbf{s}),
$$

where

$$
\left[\begin{array}{l}
e(0) \\
w(0)
\end{array}\right]
$$

is the initial-condition vector.

The system is underdamped when the roots of the transfer function characteristic equation,

$$
|\mathbf{s I}-\mathbf{A}|=(s+a)(s+b)-m_{1} m_{2}=0
$$

(where $\mathbf{I}$ is the unit matrix) are imaginary. This can occur only in 2 conditions: (1) when the coupling constants $m_{1}$ and $m_{2}$ are nonzero and one of the 2 is negative (indicating that the 2 leaky integrators are exerting a negative feedback on each other); and (2) when $(a-b)^{2}<$ $\left|4 m_{1} m_{2}\right|$.

The free response of such an underdamped system, $[r(s)=0]$, for a given initial-condition vector can be used to model the time course of the velocity storage discharge in the dark (OKAN). To this end, we can consider for simplicity the output $e(s)$ of one integrator as the system output, and change $m_{2}$ 's sign to negative to account for negative feedback (see Fig. 5). Thus,

$$
e(s)_{\text {free }}=\frac{(s+a) e(0)-m_{2} w(0)}{(s+a)(s+b)+m_{1} m_{2}} .
$$

Since the dynamic properties of the system during OKAN and during optokinetic stimulation are different (see Results), and since we do not know the $p_{2}$ value, that is, whether or not and to what extent the adaptor is charged directly by the external input, we cannot determine a priori the initial condition $w(0)$, which must thus be considered to be an unknown variable. The normalized inverse Laplace transform of equation (5) is

$$
e(t)_{\mathrm{frec}}=\frac{\exp (-T t)}{\sin (\Phi)} \cdot \sin (\Omega t+\Phi)
$$

where, by setting $w(0)=k \cdot e(0)$ and $e(0)=1$,

$$
\begin{aligned}
T & =(a+b) / 2 \\
\Omega & =1 / 2 \sqrt{4 m_{1} m_{2}-(a-b)^{2}} \\
\operatorname{tg} \Phi & =2 \Omega /\left(a-b-2 m_{2} k\right) .
\end{aligned}
$$

In case the velocity storage output were a weighed sum of the 2 leaky integrators' outputs, the right side of equation (6) would still describe the SVP time course of OKAN, with the values of $T$ and $\Omega$ determined again by equations (7) and (8), but with a different phase-shift value, $\Phi$.

For the purpose of this paper, it is also desirable to express $k$ as a function of the theoretical level of charge that the adaptor would reach at steady state if $m_{1}$ and $a$ had the same values during both $\mathrm{OKN}$ and OKAN. Indeed, there are good arguments for supposing that model parameters are not the same in the 2 conditions (see Results). The ratio, $k$, between adaptor and velocity integrator initial conditions $[w(0) / e(0)]$ can be expressed as a function of $m_{1}$ and $a$ during OKAN:

$$
k=\frac{m_{1}}{a} \cdot h,
$$

where $h$ is a constant of proportionality. When $h=1, m_{1}$ and $a$ values do not change. Instead, $h<1$ implies that during OKN, $m_{1}$ and/or the adaptor time constant $(1 / a)$ are smaller than during OKAN. The opposite is true for $h>1$. Thus, for every value of $k$ and $h$, a set of univocal $m_{1}, m_{2}, a$, and $b$ values can be computed for every observed OKAN time course.

\section{References}

Allum, J. H. J., W. Graf, J. Dichgans, and C. L. Schmidt (1976) Visualvestibular interactions in the vestibular nuclei of the goldfish. Exp. Brain Res. 26: 463-485.

Aschan, G., and M. Bergstedt (1955) The genesis of secondary nystagmus induced by vestibular stimuli. Acta Soc. Med. (Upsala) 60: 113.

Brandt, T., J. Dichgans, and W. Büchele (1974) Motion habituation: Inverted self-motion perception and optokinetic afternystagmus. Exp. Brain Res. 21: 337-352.

Buettner, U. W., U. Büttner, and V. Henn (1978) Vestibular nuclei activity in the alert monkey during sinusoidal rotation in the dark. J. Neurophysiol. 41: 1614-1628.

Buizza, A., and R. Schmid (1982) Visual-vestibular interaction in the control of eye movement: Mathematical modelling and computer simulation. Biol. Cybern. 43: 209-223.

Büttner, U., W. Waespe, and V. Henn (1976) Duration and direction of optokinetic after-nystagmus as a function of stimulus exposure time in the monkey. Arch. Psychiatr. Nervenkr. 222: 281-291.

Cazin, L., W. Precht, and J. Lannou (1980a) Firing characteristics of neurons mediating optokinetic responses to rat's vestibular neurons. Pfluegers Arch. 386: 221-230.

Cazin, L., W. Precht, and J. Lannou (1980b) Optokinetic responses of vestibular nucleus neurons in the rat. Pfluegers Arch. 384: 31-38.

Clément, G., J. H. Courjon, M. Jeannerod, and R. Schmid (1981) Unidirectional habituation of vestibulo-ocular responses by repeated rotational or optokinetic stimulations in the cat. Exp. Brain Res. 42: 34-42.

Cohen, B., V. Matsuo, and I. Raphan (1977) Quantitative analysis of the velocity characteristics of optokinetic nystagmus and optokinetic after-nystagmus. J. Physiol. (Lond.) 270: 321-344.

Cohen, B., V. Henn, T. Raphan, and D. Dennett (1981) Velocity storage, nystagmus, and visual-vestibular interactions in humans. Ann. NY Acad. Sci. 374: 421-433.

Collewijn, H. (1975) Direction-selective units in the rabbit's nucleus of the optic tract. Brain Res. 100:489-508.

Collins, W. E. (1968) Special effects of brief periods of visual fixation on nystagmus and sensations of turning. Aerospace Med. 39: 257266.

Demer, J. L. (1981) The variable gain element of the vestibulo-ocular reflex is common to the optokinetic system of the cat. Brain Res. 229: $1-13$.

Dichgans, J., C. L. Schmidt, and W. Graf (1973) Visual input improves the speedometer function of the vestibular nuclei in the goldfish. Exp. Brain Res. 18: 319-322.

Groen, J. J. (1961) Problems of the semicircular canals from a mechanico-physiological point of view. Acta Otolaryngol. (Stockh.) (Suppl. 163).

Guedry, F. E. (1974) Psychophysics of vestibular sensation. In Handbook of Sensory Physiology, vol. VI/2, H. H. Kornhuber, ed., pp. 3154, Springer-Verlag, Berlin.

Guedry, F. E., and L. S. Lauver (1961) Vestibular reactions during 
prolonged constant angular acceleration. J. Appl. Physiol. 16: 215220.

Henn, V., L. R. Young, and C. Finley (1974) Vestibular nucleus units in alert monkeys are also influenced by moving visual fields. Brain Res. 71: 144-149.

Hoffmann, K.-P., and H. P. Huber (1983) Responses to visual stimulation in single cells in the nucleus of the optic tract (NOT) during optokinetic nystagmus (OKN) in the awake cat. Soc. Neurosci. Abstr. 9: 1048.

Hoffmann, K.-P., and A. Schoppmann (1981) A quantitative analysis of the direction-specific response of neurons in the cat's nucleus of the optic tract. Exp. Brain Res. 42: 146-157.

Keller, E. L., and W. Precht (1978) Persistence of visual response in vestibular nucleus neurons in cerebellectomized cat. Exp. Brain Res. 32: 591-594.

Keller, E. L., and W. Precht (1979) Visual-vestibular responses in vestibular nuclear neurons in intact and cerebellectomized, alert cat Neuroscience 4: 1599-1613.

Koenig, E., and J. Dichgans (1981) Aftereffects of vestibular and optokinetic stimulation and their interaction. Ann. NY Acad. Sci. 374. 434-445.

Koerner, F., and P. H. Schiller (1972) The optokinetic response under open- and closed-loop conditions in monkey. Exp. Brain Res. 14. 318-330.

Leigh, R. J., D. A. Robinson, and D. S. Zee (1981) A hypothetical explanation for periodic alternating nystagmus: Instability in the optokinetic-vestibular system. Ann. NY Acad. Sci. 374: 619-635.

Lisberger, S. G., F. A. Miles, L. M. Optican, and B. B. Eighmy (1981) Optokinetic responses in monkey: Underlying mechanisms and their sensitivity to long-term adaptive changes in vestibuloocular reflex. J. Neurophysiol. 45: 869-890.

Maioli, C., and W. Precht (1984) The horizontal optokinetic nystagmus in the cat. Exp. Brain Res. 55: 494-506.

Malcolm, R., and G. Melvill-Jones (1970) A quantitative study of vestibular adaptation in humans. Acta Otolaryngol. (Stockh.) 70:126135.

Morimoto, M., K. Mizukoshi, T. Otani, S. Ikeda, Y. Katsumi, T. Sasaki, and Y. Koike (1963) On the secondary phase of nystagmus. Acta Otolaryngol. (Stockh.) (Suppl. 179): 32-41.

Neverov, V. P., J. Sterc, and J. Bures (1980) Electrophysiological correlates of the reversed postoptokinetic nystagmus in the rabbit: Activity of vestibular and floccular neurons. Brain Res. 189: 355367.

Ohm, J. (1927) Zur Augenzitterkunde. 7. Mitteilung. Der optische
Drehnachnystagmus. Albrecht Von Graefes Arch. Klin. Ophthalmol. 118: 103-117.

Oyster, C. W., E. Takahashi, and H. Collewijn (1972) Direction-selective retinal ganglion cells and control of optokinetic nystagmus in the rabbit. Vision Res. 12: 183-193.

Raphan, T., B. Cohen, and V. Matsuo (1977) A velocity storage mechanism responsible for optokinetic nystagmus (OKN), optokinetic after-nystagmus (OKAN), and vestibular nystagmus. In Control of Gaze by Brain Stem Neurons, R. Baker and A. Berthoz, eds., pp. 37-47, Flsevier, Amsterdam.

Raphan, T., V. Matsuo, and B. Cohen (1979) Velocity storage in the vestibulo-ocular reflex arc. Exp. Brain Res. 35: 229-248.

Robinson, D. A. (1977) Vestibular and optokinetic symbiosis: An example of explaining by modelling. In Control of Gaze by Brain Stem Neurons, R. Baker and A. Berthoz, eds., pp. 49-58, Elsevier, Amsterdam.

Segal, B. N., and S. Liben (1985) Modulation of human velocity storage sampled during intermittently-illuminated optokinetic stimulation. Exp. Brain Rcs. 59: 515-523.

Ter Braak, J. W. G. (1936) Untersuchungen über optokinetischen Nystagmus. Arch. Nerl. Physiol. 21: 309-376.

Waespe, W., and V. Henn (1977a) Neuronal activity in the vestibular nuclei of the alert monkey during vestibular and optokinetic stimulation. Exp. Brain Res. 27: 523-538.

Waespe, W., and V. Henn (1977b) Vestibular nuclei activity during optokinetic after-nystagmus (OKAN) in the alert monkey. Exp. Brain Res. 30: 323-330.

Waespe, W., and V. Henn (1978) Reciprocal changes in primary and secondary optokinetic after-nystagmus produced by repetitive optokinetic stimulation in the monkey. Arch. Psychiatr. Nervenkr. 225. 23-30.

Waespe, W., T. Huber, and V. Henn (1978) Dynamic changes of optokinetic after-nystagmus (OKAN) caused by brief visual fixation periods in monkeys and in man. Arch. Psychiatr. Nervenkr. 226: 110

Waespe, W., B. Cohen, and T. Raphan (1983) Role of the flocculus and paraflocculus in optokinetic nystagmus and visual-vestibular interactions: Effects of lesions. Exp. Brain Res. 50: 9-33.

Young, L. R., and C. M. Oman (1969) Model for vestibular adaptation to horizontal rotation. Aerospace Med. 40: 1076-1080.

Zee, D. S., R. D. Yee, and D. A. Robinson (1976) Optokinetic responses in labyrinthine-defective human beings. Brain Res. 113:423428 . 